\title{
Using capacitance measurements in EWOD devices to identify fluid composition and control droplet mixing
}

\author{
M.J. Schertzer, R. Ben-Mrad, P.E. Sullivan* \\ Dept. of Mechanical and Industrial Engineering, University of Toronto, 5 King's College Road, Toronto, Ontario, Canada M5S 3G8
}

\section{A R T I C L E I N F O}

\section{Article history:}

Received 10 September 2009

Received in revised form 3 December 2009

Accepted 8 December 2009

Available online 16 December 2009

\section{Keywords:}

Electrowetting on dielectric

Microfluidics

Mixing

Composition

Capacitance

Void fraction

Control

Automation

\begin{abstract}
A B S T R A C T
The use of capacitance measurements to identify the composition of droplets and monitor mixing in electrowetting on dielectric devices is examined here. Measurements were repeatable at each addressable location, with standard deviations on the order of $0.1 \mathrm{pF}$ and a two-point calibration allowed repeatable differentiation of water-methanol solutions as the capacitance was linear with concentration. Capacitance at addressable locations was monitored throughout the mixing of water-methanol solutions. It was shown analytically and experimentally that the dimensionless capacitance is approximately equal to the dimensionless dielectric constant for practical EWOD applications. The number of cycles required for complete mixing remained constant for periods of actuation between 400 and $1000 \mathrm{~ms}$ and applied voltages between 90 and $110 V_{\text {RMS }}$. Although minimizing actuation period and maximizing droplet velocity decreases mixing time, these parameters have little affect on the number of cycles necessary to achieve mixing in EWOD devices. This shows mixing efficiency in EWOD devices is better described by the number of cycles, not the time, required for full mixing.
\end{abstract}

(c) 2009 Elsevier B.V. All rights reserved.

\section{Introduction}

Over the past two decades, droplet-based microfluidic devices have been introduced as tools to increase throughput and reduce operating costs of biological protocols (e.g. [1-4]). Device platforms have been introduced to manipulate droplets by chemical [1], thermal [1], acoustic [2], and electrical [3] means. Electrowetting on dielectric (EWOD) is one of the most promising droplet-based microfluidic platforms. These devices apply asymmetric electric fields to manipulate droplets with diameters on the order of $1-2 \mathrm{~mm}$ that are confined between parallel plates separated by 50-150 $\mu \mathrm{m}(\sim 40-500 \mathrm{~nL})$ [3-5,7]. Droplets manipulated in EWOD devices have two observable phenomena: (1) asymmetric deformation of the droplet interface and (2) motion of the confined droplet. Some investigations argue that the change in the interface shape drives droplet motion (e.g. [4-6]), while others suggest that the motion is purely an electrical effect (e.g. [7,8]). Despite disagreement on the physical mechanism, these devices have demonstrated the ability to create, move, split, and mix droplets of fluid. They also have low power consumption, high reversibility, and wide applicability to different fluids [3-8]. A comprehensive review of these devices can be found in [9]. One of the proposed advantages of microfluidic systems, and EWOD devices in particular, is the ability

\footnotetext{
* Corresponding author. Tel.: +1 416978 3110; fax: +1 4169787753

E-mail address: sullivan@mie.utoronto.ca (P.E. Sullivan).
}

to increase throughput by automating protocols and running them in parallel. Monitoring EWOD devices is often performed manually, but this task must be automated for practical applications.

Most systems for automated droplet control in EWOD devices depend on either optical observation $[10,11]$, or capacitance measurements [12-15]. A system that could produce complex droplet paths in an EWOD device by actuating multiple electrodes at variable voltages was simulated in [10]. The proposed design used an optical control system to monitor droplet motion. The presence of a droplet at strategic locations can also be determined using the integrated thin-film photodetector presented in [11]. If the dielectric constant of the droplet differs from that of the surrounding medium, capacitance measurements can be used to determine the void fraction at each addressable location in an EWOD device [12-15]. Since each addressable location in these devices is essentially a parallel plate capacitor, no further fabrication would be required.

One of the earliest investigations using capacitive detection of droplets in EWOD devices was [16] which showed that capacitance measurements could detect droplets. This method has been used successfully to determine optimal usage of electrodes on EWOD devices $[12,13]$ and to create droplets of uniform size $[14,15]$. This study will examine whether capacitance measurements in EWOD devices can identify fluid composition and monitor droplet mixing.

Mixing fluids is a common problem in most microfluidic devices. On the microscale, Reynolds numbers are very low and viscous mixing dominates. Mixing in EWOD devices is commonly achieved 
(a)

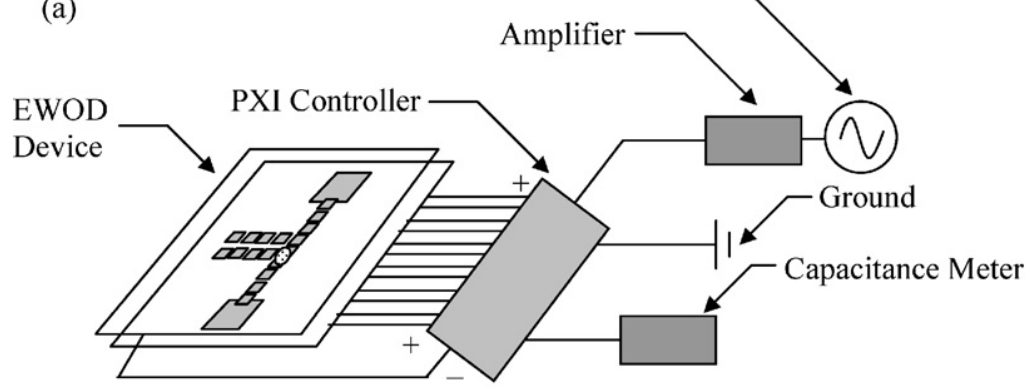

(b)

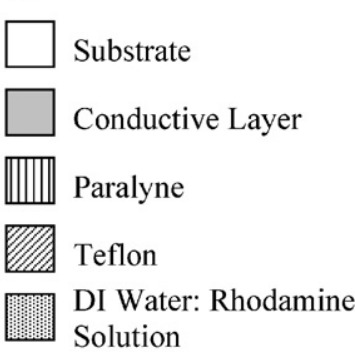

(c)
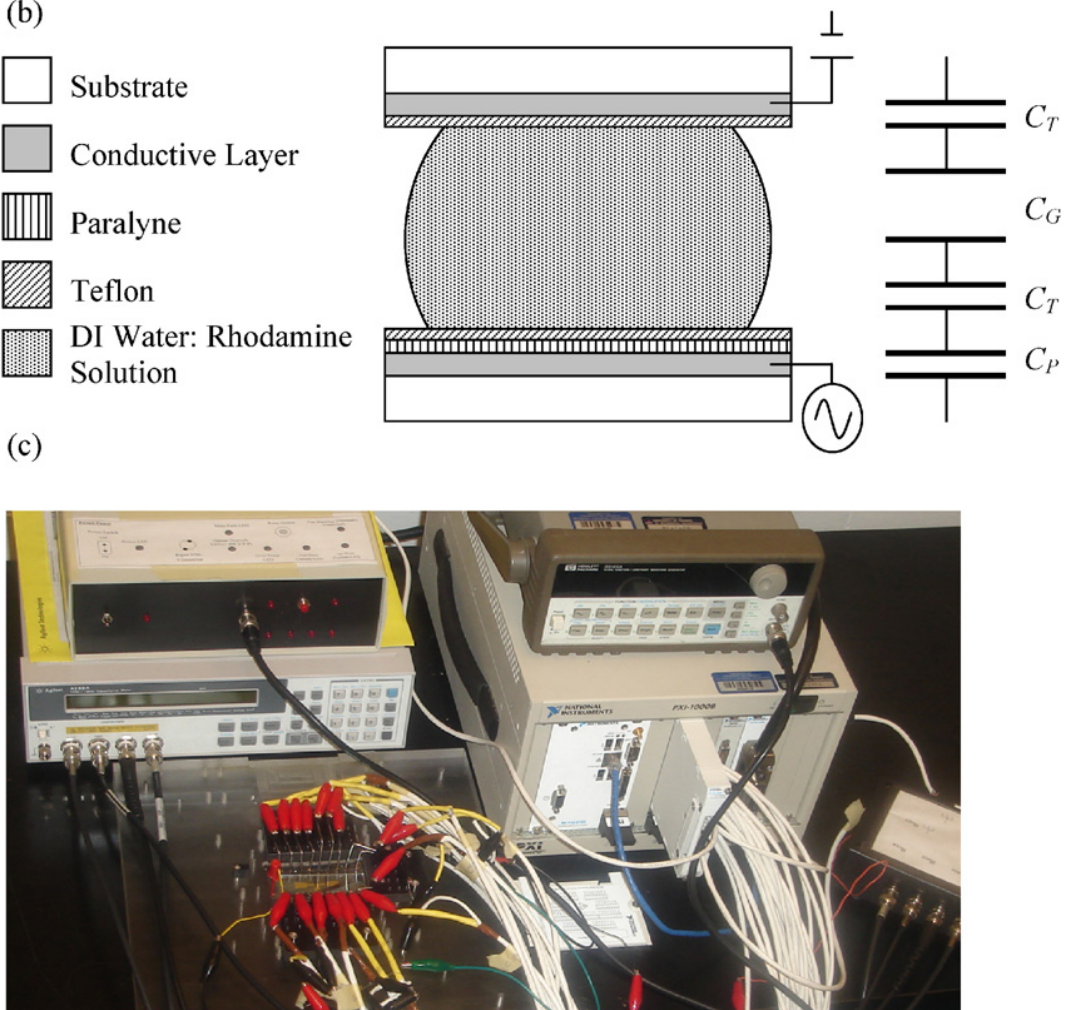

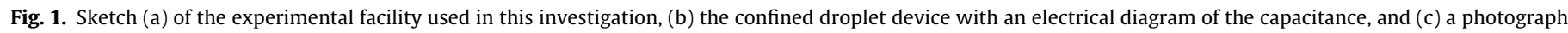
of the facility used in this investigation.

by moving droplets along a prescribed path for a specified number of cycles (i.e. [17-19]). With visualization, the mixing time required on EWOD devices was found to be dependent on the actuation frequency, the device geometry, and the path used to mix the droplets [17-19]. Mixing in droplets on the microscale is generally attributed to the folding of the fluid within the droplet as it moves along a specified path (i.e. [19]). Observations of droplet mixing in a pressure driven micromixer can be found in [20]. It has been proposed that fluid motion in EWOD devices can be enhanced and controlled by following more complex paths (i.e. $[17,18]$ ) or imposing a time dependant rigid-body rotation [21]. It would be advantageous to be able to monitor droplet mixing without intrusive techniques or optical access to the device.

The objective of this investigation is to use capacitance measurements in EWOD devices to identify fluids of differing composition and to monitor droplet mixing without requiring optical access to the chip, modification of the droplet, or additional fabrication.

\section{Experimental facility}

The experimental facility used in this investigation consisted of the EWOD device and the electrical control system (Fig. 1a).
The EWOD device was made up of two silica glass slides. The upper and lower plates were approximately $25 \mathrm{~mm} \times 75 \mathrm{~mm}$ and $75 \mathrm{~mm} \times 75 \mathrm{~mm}$, respectively. Both plates were cleaned using an organic solvent, Piranha, before fabrication. The lower plate was coated with a conductive layer made up of chromium and gold. This conductive layer was patterned into an array of $2 \mathrm{~mm} \times 2 \mathrm{~mm}$ electrodes separated by a gap of approximately $60 \mu \mathrm{m}$. The bottom plate was also coated with a dielectric layer of Parylene. A Teflon layer was then added to increase the hydrophobicity of the surface. The slides used for the upper plate were purchased with a pre-existing ITO coating. A Teflon layer was also added to this plate. The thickness of the chromium, gold, ITO, Parylene, and the Teflon layers were approximately $10 \mathrm{~nm}, 100 \mathrm{~nm}, 150 \mathrm{~nm}, 1.5 \mu \mathrm{m}$, and $50 \mathrm{~nm}$ respectively. The Parylene and Teflon layers above the electrical bond pads were manually scratched away to provide good electrical contact.

The spacing between the two substrates is achieved using one piece of double sided $3 \mathrm{M}$ Scotch tape with a nominal thickness of approximately $95 \mu \mathrm{m}$ [22]. The confined droplets in this investigation are solutions of DI water and methanol at various concentrations (Fig. 1b). Droplets of known volumetric concentration were deposited on the bottom substrate using a pipette 
before adding the upper plate. The volume of fluid in droplet detection and mixing experiments was 1300 and $650 \mathrm{~nL}$, respectively. The uncertainty in the pipette measurements was $1 \%$. The mixing experiments used droplets of deionized water and a 75\% methanol solution. Pure methanol was not used in these experiments as it evaporated during mixing.

Selective application of the electric field in the EWOD device was achieved with a control system consisting of a National Instruments PXI system, an Agilent 33120A signal generator, a custom amplifier, and an Agilent 4288A capacitance meter. The electrical signal was controlled by the PXI system: a PXI controller (PXI 8195), a matrix-switching device (PXI 2529) capable of controlling four inputs with up to 32 outputs, and a PXI GPIB controller. The inputs were the AC source and the capacitance meter. The output channels were connected to bond pads for each addressable location on the EWOD device using a custom built fixture (Fig. 1c). The electrodes were connected to the high voltage source for $350 \mathrm{~ms}$, and the capacitance meter for 50-650 ms. The sequencing of these connections was programmed using Labview Real Time 8.2. The AC source voltage in this investigation was generated using an Agilent signal generator and a custom built amplifier. The actuation frequency in this investigation was held fixed at $10 \mathrm{kHz}$ and the actuation voltage was between 90 and $110 \mathrm{~V}_{\mathrm{RMS}}$. Control of the capacitance meter was achieved using the GPIB controller in the PXI system described above. The test voltage for the capacitance meter was $1 \mathrm{~V}_{\mathrm{RMS}}$ at a frequency of $1 \mathrm{MHz}$. The accuracy of this device is given as $0.08 \%$ for these parameters. For the magnitude of capacitance examined here, this led to a resolution of approximately $0.20 \mathrm{pF}$.

\section{Results and discussion}

If each addressable position in the EWOD device is modeled as a number of parallel plate capacitors in series (Fig. 1b), then the capacitance in each measurement volume is given by

$C_{F i}=\frac{\varepsilon_{0} \varepsilon_{T} \varepsilon_{P} \varepsilon_{F i} A}{\varepsilon_{F i}\left(2 \varepsilon_{P} t_{T}+\varepsilon_{T} t_{P}\right)+\varepsilon_{T} \varepsilon_{P} t_{G}}$,

where $\varepsilon_{0}$ is the permittivity of free space, $\varepsilon$ is a dielectric constant, $A$ is the area of the electrode, $t$ is the material thickness, and the subscripts $T, P, G$ and $F i$ denote the Teflon layer, the Paralyne layer, the gap between the substrates, and the fluid in the measurement volume respectively. In order to understand how capacitance measurements in EWOD devices change with droplet composition, it is useful to derive the difference between two capacitance measurements. Using (1), it can be shown that the difference between the capacitance and a reference capacitance is

$C_{F 1}-C_{F 2}=\frac{\varepsilon_{0} A}{t_{G}} \beta\left(\varepsilon_{F 1}-\varepsilon_{F 2}\right)$,

where
Table 1

Material Properties.

\begin{tabular}{lc}
\hline Parameter (unit) & Value \\
\hline Dielectric constants: & \\
Air & 1.0 \\
Methanol & 33.0 \\
$75 \%$ methanol & 44.9 \\
Water & 80.4 \\
Material thickness: & \\
Teflon $(\mathrm{nm})$ & 10 \\
Parylene $(\mu \mathrm{m})$ & 1.5 \\
Gap $(\mu \mathrm{m})$ & 100 \\
\hline
\end{tabular}

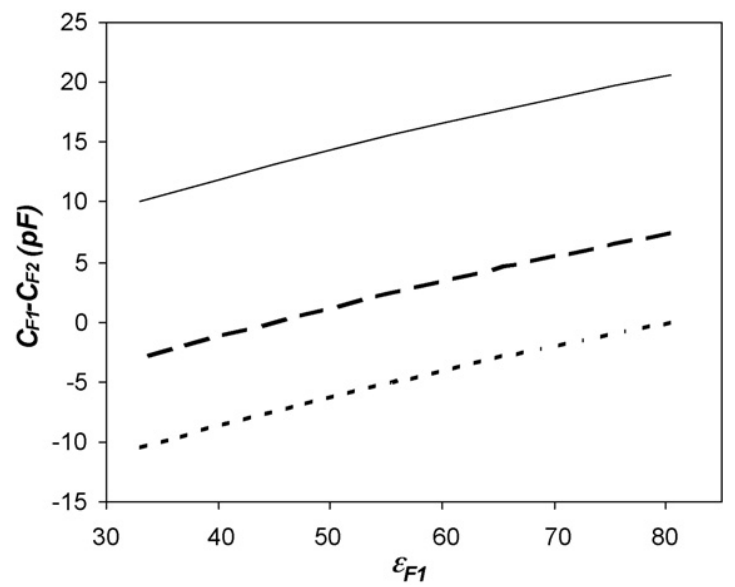

Fig. 2. Predicted difference in capacitance with a reference capacitance of air (solid line), $75 \%$ methanol (dashed line) and water (dotted line).

The predicted difference in capacitance is shown in Fig. 2 for reference capacitances of air, $75 \%$ methanol, and water. In all cases, the increase in the difference between the capacitance and the reference capacitance with $\varepsilon_{F 1}$ is approximately linear. The slopes of a linear fit in all cases agree to within $0.4 \%$. This shows that the difference in capacitance is approximately linear in the range of interest in EWOD devices. It is important to realize that the value of $\beta$ affects the sensitivity of $C_{F 1}-C_{F 2}$. For example, as $t_{P} \rightarrow 0$ then $\beta \rightarrow 1$ and $C_{F 1}-C_{F 2} \rightarrow \varepsilon_{0} A\left(\varepsilon_{F 1}-\varepsilon_{F 2}\right) / t_{G}$. Thus, the EWOD device can be designed to increase the sensitivity of capacitance measurements.

Since the difference in the capacitance is approximately linear with $\left(\varepsilon_{F 1}-\varepsilon_{F 2}\right)$, the dimensionless capacitance $C^{*}=\left(C_{F}-\right.$ $\left.C_{F 2}\right) /\left(C_{F 1}-C_{F 2}\right)$ is approximately equal to the dimensionless dielectric constant $\varepsilon^{*}=\left(\varepsilon_{F}-\varepsilon_{F 2}\right) /\left(\varepsilon_{F 1}-\varepsilon_{F 2}\right)$. Here, the subscript $F$, refers to the fluid in the measurement volume and the subscripts $F 1$, and $F 2$, refer to two reference fluids respectively. This

$\beta=\frac{1}{\left(4 \varepsilon_{F 1} \varepsilon_{F 2} / \varepsilon_{T}^{2}\right)\left(t_{T}^{2} / t_{G}^{2}\right)+\left(4 \varepsilon_{F 1} \varepsilon_{F 2} / \varepsilon_{P} \varepsilon_{T}\right)\left(t_{T} t_{P} / t_{G}^{2}\right)+\left(\varepsilon_{F 1} \varepsilon_{F 2} / \varepsilon_{P}^{2}\right)\left(t_{P}^{2} / t_{G}^{2}\right)+\left(2\left(\varepsilon_{F 1}+\varepsilon_{F 2}\right) / \varepsilon_{T}\right)\left(t_{T} / t_{G}\right)+\left(\left(\varepsilon_{F 1}+\varepsilon_{F 2}\right) / \varepsilon_{P}\right)\left(t_{P} / t_{G}\right)+1}$

and the subscripts $F 1$ and $F 2$ denote the fluid being examined and a reference fluid, respectively. As the fluid within the measurement volume (F1) changes, $\varepsilon_{F 1}$ changes as well. Since $\varepsilon_{F 1}$ exists in $\beta$, it introduces non-linearity into (2). An order of magnitude analysis can be used to understand the extent of this non-linearity. In most cases, $\varepsilon_{F 1}$ and $\varepsilon_{F 2}$ will be an order of magnitude larger than $\varepsilon_{P}$ and $\varepsilon_{T}$ (Table 1 ). In the devices tested here $t_{G}$ was four orders of magnitude greater than $t_{T}$ and two orders of magnitude greater than $t_{P}$ (Table 1 ). The highest order term in the denominator of $\beta$ is 1 . If terms in the denominator of $\beta$ smaller than $10^{-2}$ are neglected, then:

$\beta \approx \frac{1}{\left(\varepsilon_{F 1} \varepsilon_{F 2} / \varepsilon_{P}^{2}\right)\left(t_{P}^{2} / t_{G}^{2}\right)+\left(\left(\varepsilon_{F 1}+\varepsilon_{F 2}\right) / \varepsilon_{P}\right)\left(t_{P} / t_{G}\right)+1}$. relationship is shown in Fig. 3. For the cases examined here $F 1$ was always water and $F 2$ was air, methanol, or $75 \%$ methanol by volume. The difference between $C^{*}$ and $\varepsilon^{*}$ decreases with $\left(\varepsilon_{F 1}-\varepsilon_{F 2}\right)$ because the non-linearity in $(2)$ decreases with $\left(\varepsilon_{F 1}-\varepsilon_{F 2}\right)$. Therefore, the difference in the dielectric constants of the reference fluids should be minimized in order to identify fluids in EWOD devices. As such, the reference fluids should be those with the highest and lowest dielectric constants in an application. When water and methanol are used as the reference fluids, the value of $C^{*}$ differs from that of $\varepsilon^{*}$ by an average of 0.03 and a maximum of 0.05 . When water and $75 \%$ methanol were used, the average and maximum errors decrease to 0.02 and 0.04 respectively. 


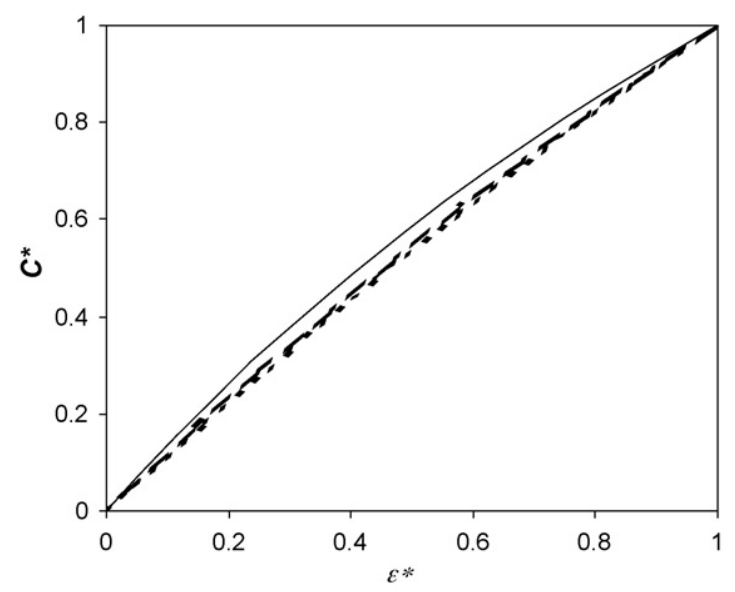

Fig. 3. Comparison of dimensionless capacitance and dielectric constants when $F 1$ is water and an F2 is air (solid line), methanol (dashed line), and 75\% methanol (dotted line).

Multiple capacitance measurements for a water droplet actuated between three electrodes in an EWOD device were taken to confirm repeatability; the distribution $(G(T))$ is shown in Fig. 4. Although the standard deviations in each measurement volume range between 0.05 and $0.08 \mathrm{pF}$, the standard deviation of the entire population is approximately $0.45 \mathrm{pF}$. This suggests that although the capacitance measurements are repeatable at every addressable location, measurement calibration is required in order to compare data from different locations.

The type of calibration required can be determined by examining the measured capacitance as a function of the dielectric constant (Fig. 5). Here, each point on the curve is an average of 30 measurements distributed over three electrodes. As expected, the capacitance of the mixture increases linearly with $\varepsilon_{F}$. The slope of the change in capacitance is approximately $0.08 \mathrm{pF}$ for every unit change in the dielectric constant. This is approximately $0.04 \mathrm{pF}$ per percentage decrease in methanol concentration. Since the experimental resolution is on the order of $0.2 \mathrm{pF}$, this suggests that it is possible to detect changes in

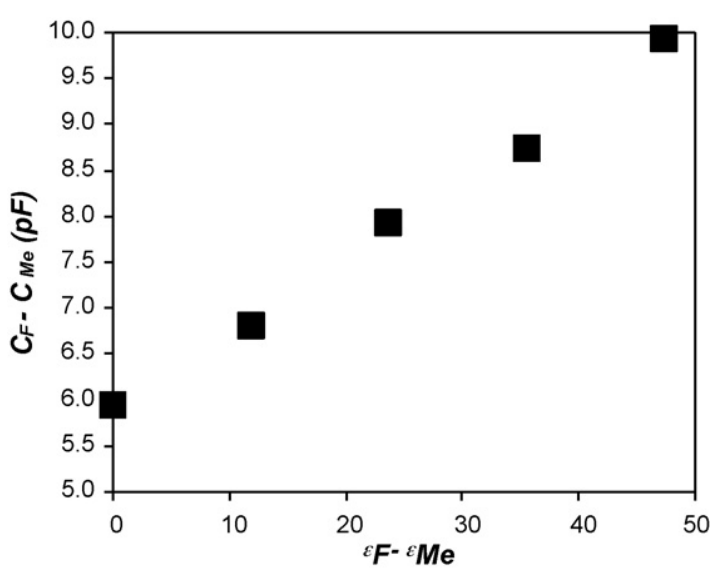

Fig. 5. Average $\Delta C$ as a function of the $\Delta \varepsilon$.

composition on the order of $5 \%$ methanol by volume. Because the change in capacitance is linear with concentration, the relative capacitance at each electrode was found using a two-point calibration. After performing the calibration, the standard deviation of the population of measurements for each solution was below $0.13 \mathrm{pF}$ (Fig. 6). This is closer to the standard deviation on each channel and below the resolution of measurements. This suggests it is possible to identify the composition of solutions on EWOD devices and compare data from any addressable location.

Another possible issue with comparing capacitance measurements of droplets in EWOD devices is the effect that the droplet radius has on the measurements. As droplets move through EWOD devices, they are susceptible to evaporation. This will change their cross-sectional area and could affect the capacitance measurements. The droplet radius was varied between 650 and $1300 \mathrm{~nL}$ in this investigation. Water and $75 \%$ methanol droplets of various volumes were actuated back and forth over four electrodes a total of 40 times. The average capacitance for each radius was found to agree with the average of the total population to within 5\% (Fig. 7). The droplet radius has little effect on the capacitance measurements because the capacitance measurement is a function of the area of
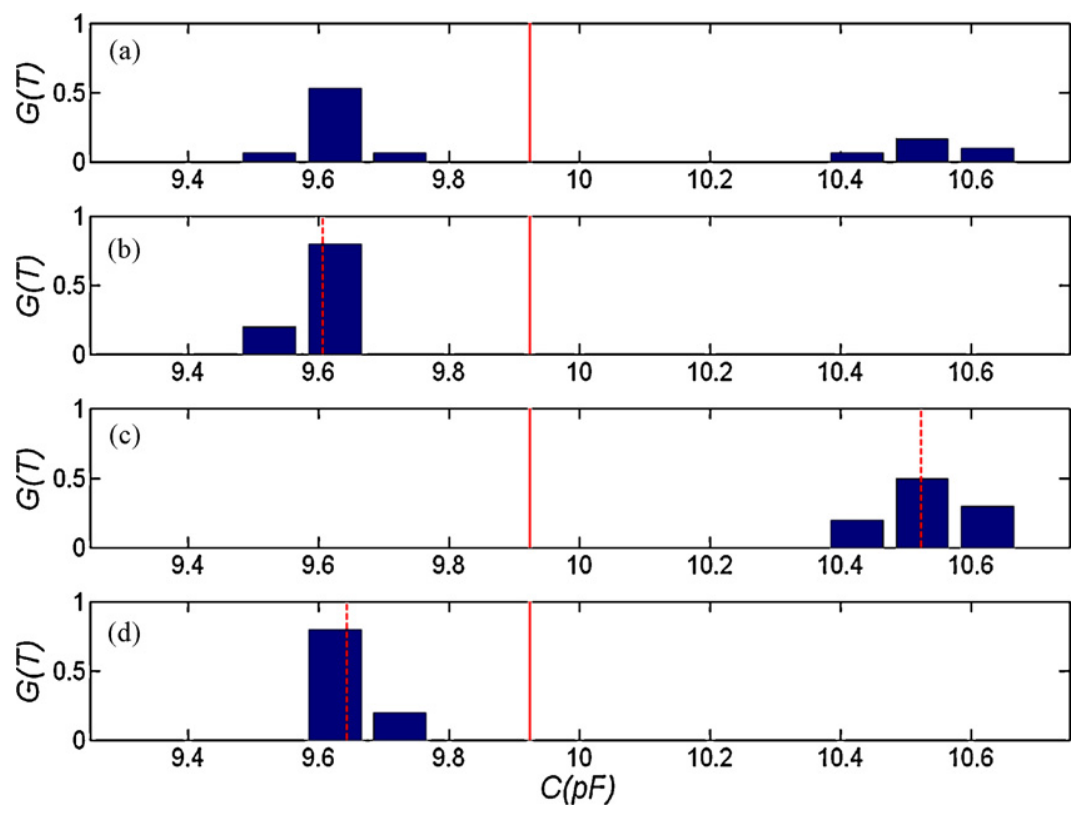

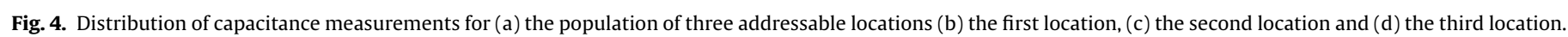
Also shown are the average of the population (solid line) and the averages of the individual locations (dashed lines). 

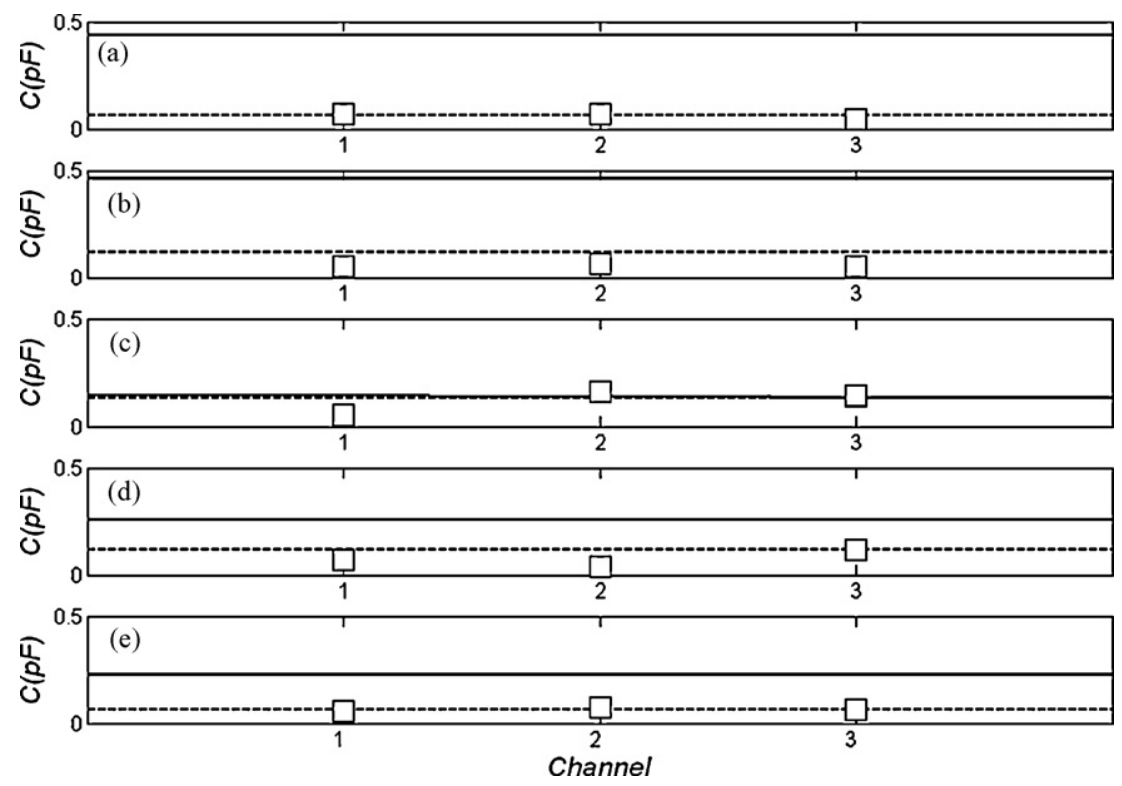

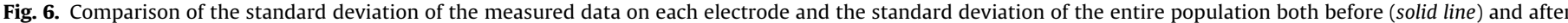
(dashed line) calibration for solutions with methanol concentration of (a) $0 \%$, (b) $25 \%$, (c) $50 \%$, (d) $75 \%$, and (e) $100 \%$.

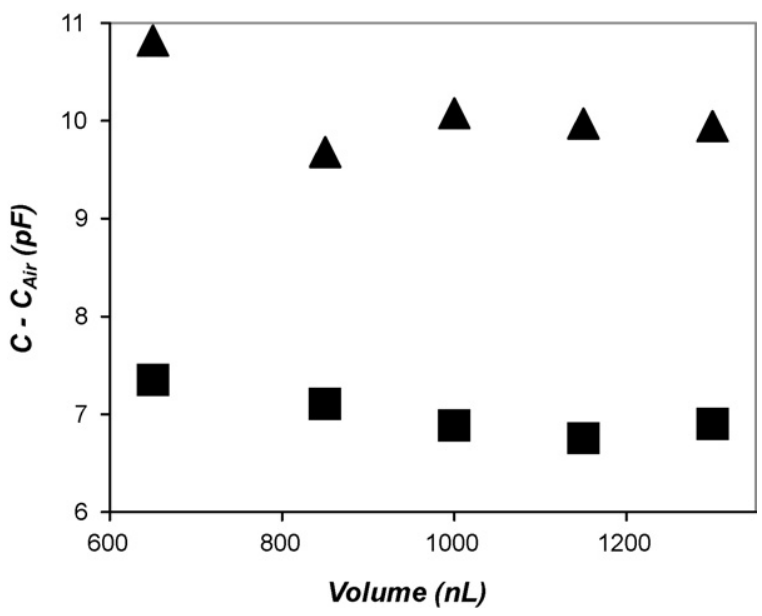

Fig. 7. Average $\Delta C$ as a function of droplet volume for water (triangle) and $75 \%$ methanol by volume (square).

the electrode so long as the droplet fills the entire measurement volume. This has been suggested by [14].

The measured and predicted difference between the droplet's capacitance and that of methanol are presented in Fig. 8. The measured and predicted slopes are 0.08 and $0.22 \mathrm{pF}$ for every unit change in the dielectric constant. This discrepancy shows that the simple series capacitance model presented here does not wholly capture the physics. The disagreement may be the result of parasitic capacitance, or the capacitance caused by the build up of charge at the droplet interface. Although the model presented here does not predict the value of the measured data, it does predict the shape. Since the measured data is still approximately linear, $C^{*}$ still provides a valid approximation of $\varepsilon^{*}$ (Fig. 9). As with the predicted case, the accuracy of approximation increases as $\left(\varepsilon_{F 1}-\varepsilon_{F 2}\right)$ decreases. From this, $C^{*}$ is a reasonable approximation of $\varepsilon^{*}$ for practical cases, despite the limitations of (2).

The change in dimensionless capacitance $C^{*}=(C-$ $\left.C_{M e 75}\right) /\left(C_{W}-C_{M e 75}\right)$ during the mixing of water and $75 \%$ methanol droplets is shown in Fig. 10. Data for the motion of $1300 \mathrm{~nL}$ droplets of water and $75 \%$ methanol are also provided to show that the value in these control cases does not change due to a change in

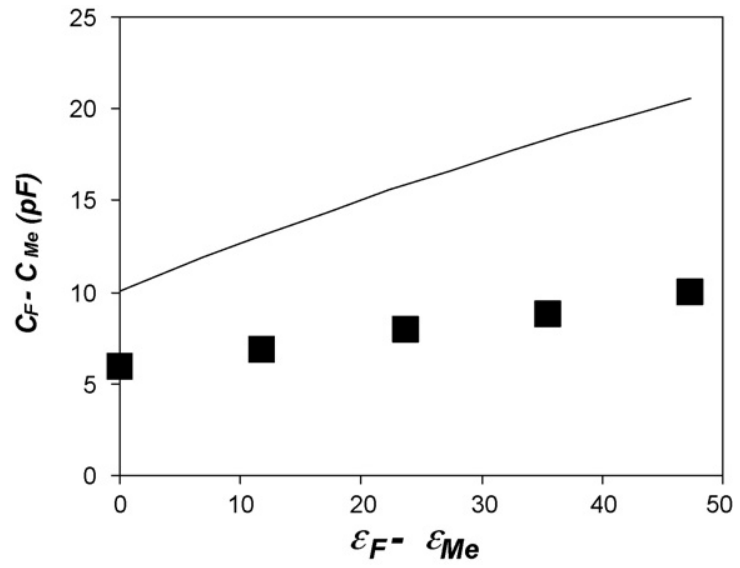

Fig. 8. Comparison of the measured (square) and predicted (line) $\Delta C$ as a function of the $\Delta \varepsilon$.

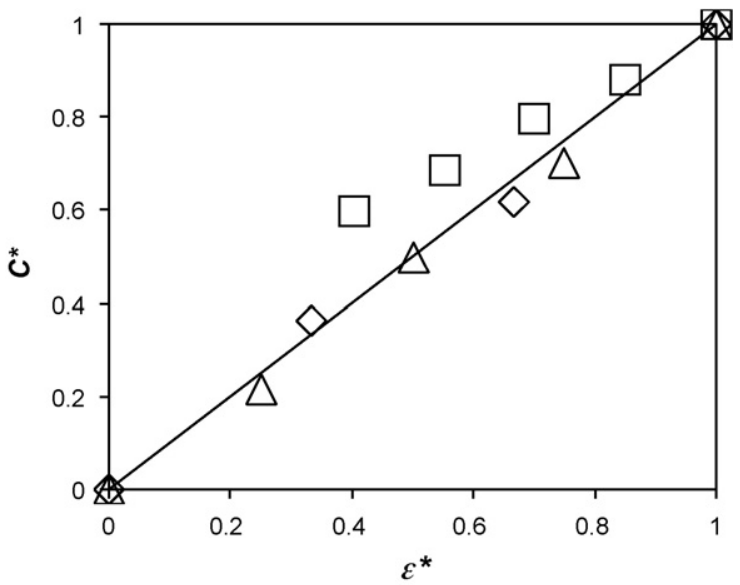

Fig. 9. Comparison of the measured values of $C^{*}$ with water as $F 1$ and $F 2$ as air (square), methanol (triangle) and $75 \%$ methanol (diamond). A solid line with a slope of 1 is given to show where $C^{*}=\varepsilon^{*}$. 


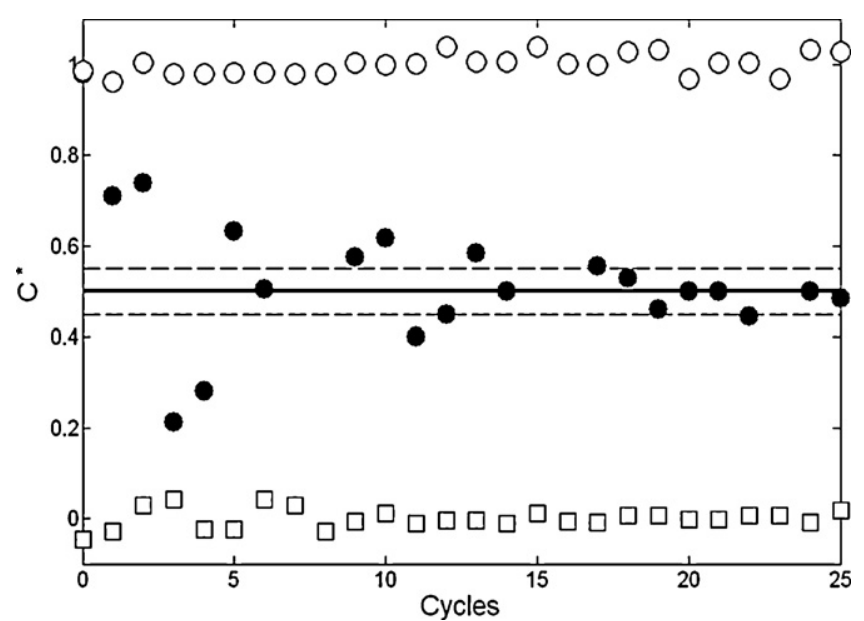

Fig. 10. Dimensionless capacitance measurements from all electrodes during the mixing of water and $75 \%$ methanol (closed circle). Control cases of water (open circle) and $75 \%$ methanol (open square), and the expected capacitance (solid line) and the experimental resolution (dashed line) are also shown.

droplet volume, or a build up in charge at the interface. Initially, the capacitance measurements in the mixed droplet range between the capacitance of water and the $75 \%$ methanol solution. As the droplets are mixed, the capacitance approaches the expected capacitance of a $37.5 \%$ methanol solution. Since the capacitance for all electrodes converges in Fig. 9, mixing can be monitored in EWOD devices by measuring the capacitance at a single location along the mixing path.

The dimensionless capacitance for a single electrode is shown in Fig. 11a. The capacitance is initially close to that of water and approaches a steady state value over time. If a single electrode is being used, uncalibrated data could monitor droplet mixing. Typical uncalibrated mixing data for a single location is shown in Fig. 11b. The first capacitance measurement from this electrode is approximately 9.3 pF and oscillates about 8.35 pF once mixing is completed. Eliminating the calibration decreases cycle time; however, more motion cycles are necessary to ensure complete mixing, as the target capacitance is unknown.

In this investigation, the droplet is considered fully mixed when the capacitance remains at the target capacitance plus or minus the experimental resolution $(\sim 0.2 \mathrm{pF})$. The capacitance measurements in Figs. 10 and 11 come to steady state at approximately 15 cycles, or $6 \mathrm{~s}$. The number of cycles is similar to that reported in [18], and the time required for mixing is similar to that reported by [17] for a device with aspect ratios between 0.33 and 0.5 . However, the aspect ratio here is 0.05 and [17] found that mixing time increased with aspect ratio below 0.4 . It is unclear if mixing time or number of cycles gives a more appropriate characterization of mixing efficiency in EWOD devices. Mixing time consists of two components (1) the time the droplet is at rest between actuations and (2) the time the droplet takes to travel from one electrode to another. The effect of these parameters will be examined to determine if mixing time or number of cycles gives a better characterization of mixing efficiency in EWOD devices.

Capacitance was used to examine the relative importance of passive diffusion while two droplets were mixed on an EWOD device. This was accomplished by varying the actuation period between 400 and 1000 ms along the EWOD mixer. As the period increases, the droplet sits idle on each electrode for a longer period of time, allowing more time for passive diffusion to take place. Typical results for periods of 400, 600, and 1000 ms are presented in Fig. 12. The capacitance measurements in these three cases are similar, with the droplet achieving steady state at roughly 15 cycles. Viscous mixing inside the droplet dominates over diffusion here since the number of cycles required for mixing remained consistent over the range of periods tested. This is consistent with the literature (i.e. $[19,20])$ and suggests that EWOD mixers should operate at the minimum period required for repeatable droplet mixing. It also shows that total mixing time is not a suitable parameter to define mixing efficiency in EWOD devices. It is not yet clear if transit time or number of cycles is a more appropriate parameter.

Capacitance measurements were also used to examine the effect of actuation voltage on mixing effectiveness in EWOD devices. The actuation voltage in the device was increased from 90 to $110 \mathrm{~V}_{\mathrm{RMS}}$. Increasing the voltage increases the droplet velocity provided that
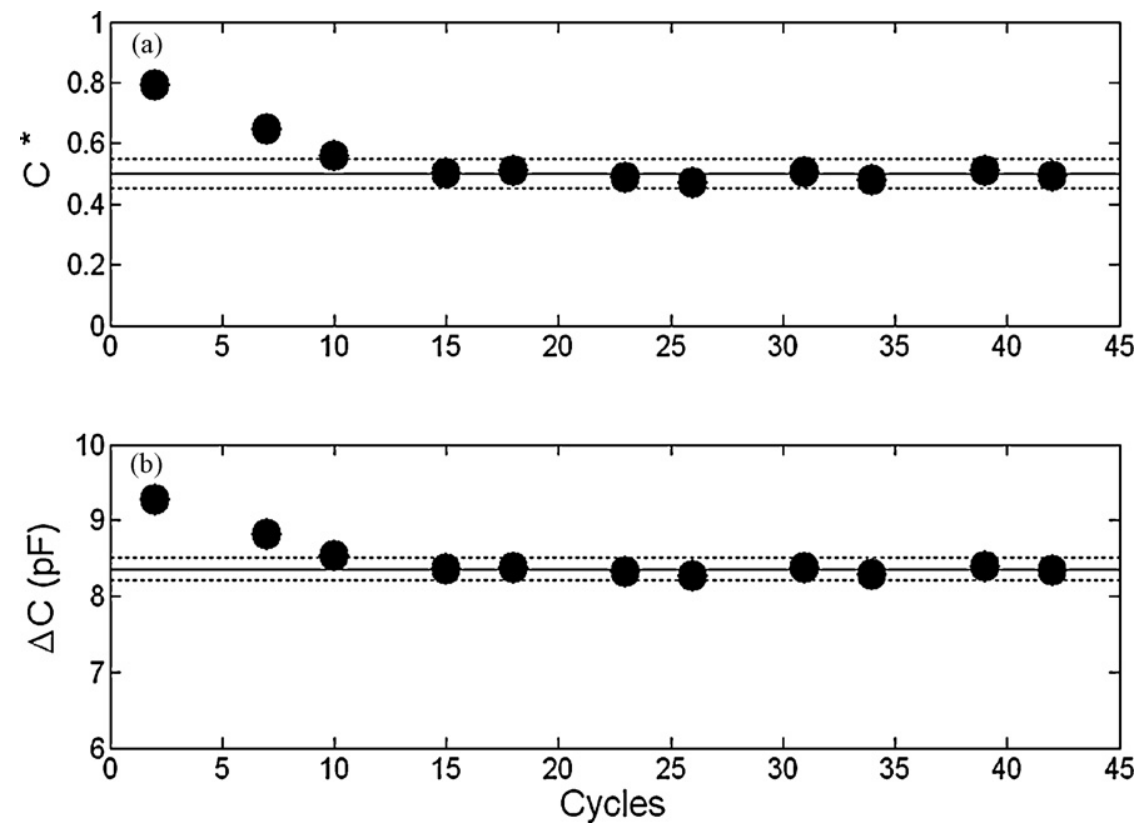

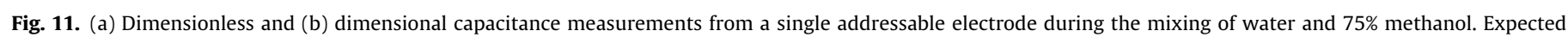
capacitance (solid line) and the experimental resolution (dashed line) are also shown. 

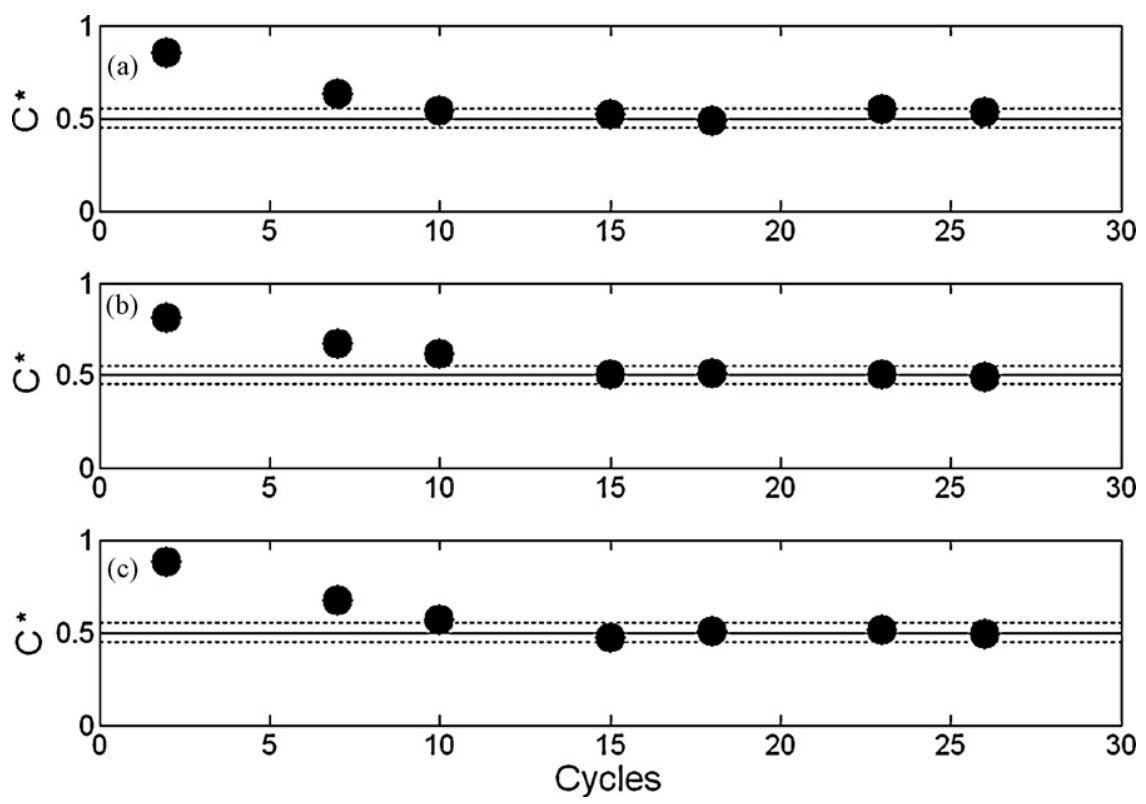

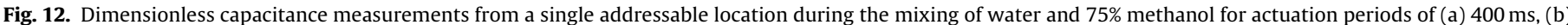
$600 \mathrm{~ms}$, and (c) $1000 \mathrm{~ms}$. Expected capacitance (solid line) and the experimental resolution (dashed line) are also shown.
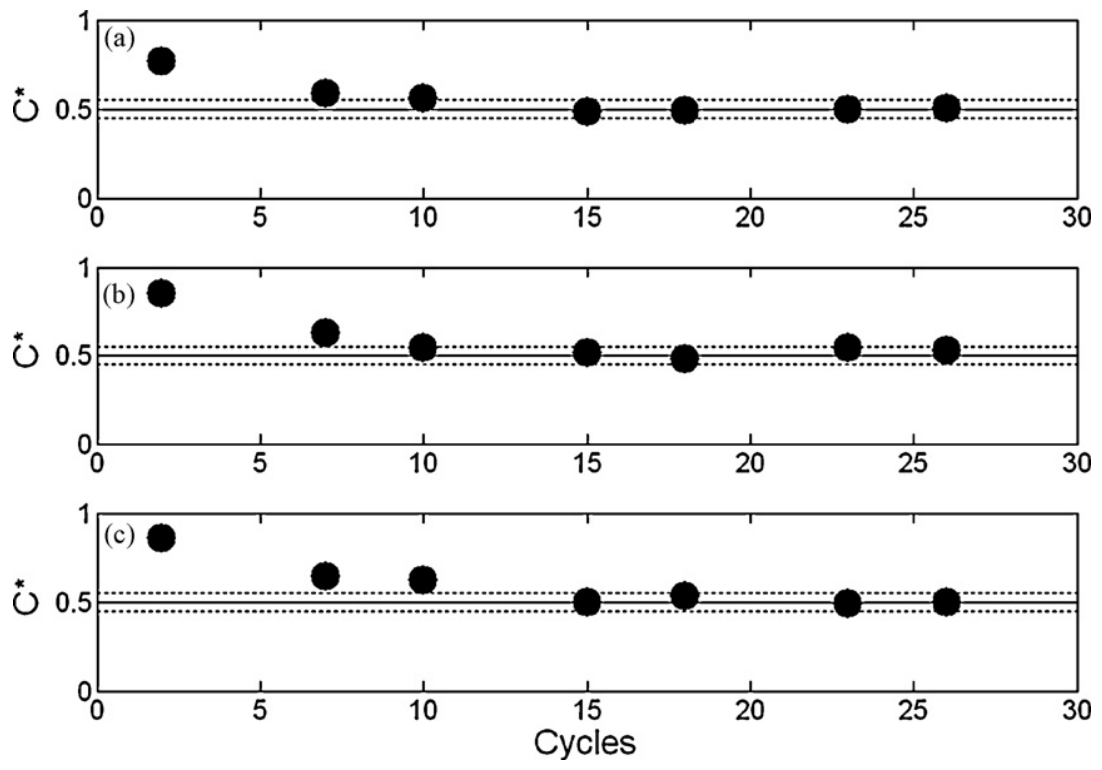

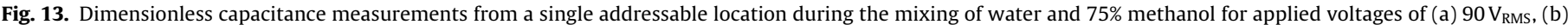
$100 \mathrm{~V}_{\mathrm{RMS}}$, and (c) $110 \mathrm{~V}_{\mathrm{RMS}}$. Expected capacitance (solid line) and the experimental resolution (dashed line) are also shown.

contact angle has not been saturated [5]. Although droplet velocity was not measured, from inspection the velocity did increase from 90 to $110 \mathrm{~V}_{\mathrm{RMS}}$. At $90 \mathrm{~V}_{\mathrm{RMS}}$, the droplet took the majority of the actuation time to move from one electrode to the next, but this time reduced rapidly at higher voltages. Droplets did not repeatably move the length of the electrode for voltages below $90 \mathrm{~V}$. Contact angle saturation occurs above $130 \mathrm{~V}$ with $0.1 \mathrm{M}$ potassium chloride for a dielectric layer thickness of $2 \mu \mathrm{m}$ [23]. If the onset of contact angle saturation is similar in this investigation, droplet velocity will increase from 90 to $110 \mathrm{~V}_{\mathrm{RMS}}$. The observed increase in velocity corresponds to a decrease in the droplet transit time. Despite the difference in transit time, the evolution of capacitance with the number of cycles was similar for all voltages examined here, with the droplet achieving steady state at roughly 15 cycles (Fig. 13). Since there is little effect of applied voltage over this range, an increase in the velocity of the droplet does not increase the effec- tiveness of mixing in EWOD devices. This is expected for viscosity dominated mixing at the microscale. This also shows that mixing effectiveness in EWOD devices is best described by the number of cycles required, not the transit time for the droplet.

\section{Conclusions}

Capacitance measurements can determine the composition of a droplet at a specific addressable location and monitor the mixing process on an EWOD device. Dimensionless capacitance for EWOD devices was analytically shown to be approximately equal to the dimensionless dielectric constant. This result was verified experimentally. Since the change in the capacitance was approximately linear, a two-point calibration of the capacitance measurements at each addressable position allowed for comparison of data at every position within the device. 
Capacitance measurements are a useful tool to determine the state of droplet mixing in EWOD devices. The capacitance was initially near the values of the first droplet compositions and proceeded to the expected capacitance of the fully mixed solution. Calibrated and uncalibrated capacitance measurements at a single addressable location were sufficient to determine when the solution was fully mixed. Capacitance measurements were taken throughout mixing for actuation periods between 400 and $1000 \mathrm{~ms}$, and voltages between 90 and $110 \mathrm{~V}_{\mathrm{RMS}}$. It was shown that increasing the idle time between actuations, and the transit time of the droplet during mixing had little effect on the number of cycles required for full mixing. This shows mixing efficiency in EWOD devices is best described by the number of cycles, not the time, required for full mixing.

\section{References}

[1] F. Brochard, Motions of droplets on solid surfaces induced by chemical or thermal gradients, Langmuir 5 (1989) 432-438.

[2] A. Wixforth, C. Strobl, C. Gauer, A. Toegl, J. Scriba, Z.V. Guttenberg, Acoustic manipulation of small droplets, Analytical and Bioanalytical Chemistry 379 (2004) 289-991.

[3] M. Washizu, Electrostatic actuation of liquid droplets for microreactor applications, IEEE Transactions on Industry Applications 34 (4) (1998) 732-737.

[4] S. Cho, H. Moon, C.J. Kim, Creating, transporting, cutting, and merging liquid droplets by electrowetting-based actuation for digital microfluidic circuits, Journal of Microelectromechanical Systems 12 (2003) 70-79.

[5] H. Ren, R. Fair, M. Pollack, E. Shaughnessy, Dynamics of electro-wetting droplet transport, Sensors and Actuators B 87 (2002) 201-206.

[6] S.W. Walker, B. Shapiro, Modeling the fluid dynamics of electro-wetting on dielectric (EWOD), Journal of Microelectromechanical Systems (2006) 986-1000.

[7] D. Chatterjee, B. Hetayothin, A.R. Wheeler, D. King, R.L. Garrell, Droplet-based microfluidics with non-aqueous solvents and solutions, Lab on a Chip (2006) 199-206.

[8] T.B. Jones, On the relationship of dielectrophoresis and electrowetting, Langmuir (2002) 4437-4443.

[9] J. Berthier, Microdrops and Digital Microfluidics, William Andrew Pub., Norwich, NY, 2008.

[10] S. Walker, B. Shapiro, A control method for steering individual particles inside liquid droplets actuated by electrowetting, Lab on a chip (2005) 1404-1407.

[11] L. Luan, R.D. Evans, N.M. Jokerst, R.B. Fair, Integrated optical sensor in a digital microfluidic platform, IEEE Sensors (2008) 628-634.

[12] F. Su, S. Ozev, K. Chakrabarty, Ensuring the operational health of droplet-based microelectrofluidic biosensor systems, IEEE Sensors Journal (2005) 763-773.

[13] F. Su, S. Ozev, K. Chakrabarty, Concurrent testing of digital microfluidics-based biochips, ACM Transactions on Design Automation of Electronic Systems (2006) 442-464.

[14] H. Ren, R.B. Fair, M.G. Pollack, Automated on-chip droplet dispensing with volume control by electro-wetting actuation and capacitance metering, Sensors and Actuators B (2004) 319-327.

[15] J. Gong, C.J. Kim, All-electronic droplet generation on-chip with real-time feedback control for EWOD digital microfluidics, Lab on a Chip (2008) 898-906.

[16] M. Pollack, Electrowetting-based microactuation of droplets for digital microfluidics, Ph.D. Thesis, Duke University, North Carolina, 1999.

[17] P. Paik, V.K. Pamula, R.B. Fair, Rapid droplet mixers for digital microfluidic systems, Lab on a Chip (2003) 253-259.
[18] Y. Fouillet, D. Jary, C. Chabrol, P. Claustre, C. Peponnet, Digital microfluidic design and optimization of classic and new fluidic functions for lab on a chip systems, Microfluidics and Nanofluidics (2008) 159-165.

[19] J. Fowler, H. Moon, C.J. Kim, Enhancement of mixing by droplet-based microfluidics, Technical Digest, in: MEMS 2002 IEEE International Conference. Fifteenth IEEE International Conference on Micro Electro Mechanical Systems (Cat. No.02CH37266), 2002, pp. 97-100.

[20] Z.B. Stone, H.A. Stone, Imaging and quantifying mixing in a model droplet micromixer, Physics of Fluids (2005) 1-11.

[21] R. Chabreyrie, D. Vainchtein, C. Chandre, P. Singh, N. Aubry, Tailored mixing inside a translating droplet, Physical Review (2008) 1-4.

[22] M.J. Schertzer, S.I. Gubarenko, R. Ben-Mrad, P.E. Sullivan, An empirically validated model of the pressure within a droplet confined between plates at equilibrium for low bond numbers, Experiments in Fluids, published online November 5, 2009, doi:10.1007/s00348-009-0773-8, 2009.

[23] A. Quinn, R. Sedev, J. Ralston, Influence of the electric double layer in electrowetting, Journal of Physical Chemistry 107 (2003) 1163-1169.

\section{Biographies}

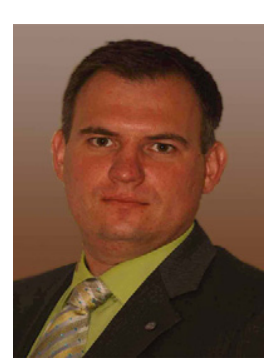

M.J. Schertzer received the B.Eng.Mgt and M.A.Sc. degrees in Mechanical Engineering from McMaster University, Hamilton, ON, in 2003 and 2005, respectively, and is currently working on the completion of the Ph.D. degree in Mechanical and Industrial Engineering at the University of Toronto, Toronto, ON. His areas of interest include microfluidics and two-phase heat transfer.

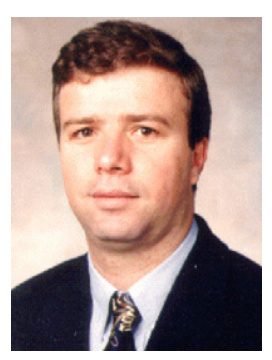

R. Ben-Mrad received in 1986 the B.Sc. from the Pennsylvania State University in Mechanical Engineering, the M.A.Sc. in 1988 and the Ph.D. in 1994 from the University of Michigan in Mechanical Engineering. He also received the M.Sc. in 1991 in Electrical Engineering from the University of Michigan. He has been since 1997 with the Department of Mechanical and Industrial Engineering at the University of Toronto. He is currently a Professor. His research interests include MEMS fabrication and design, microfluidics, design of piezoceramic actuated linear motors, and development and characterization of smart materials-based sensors and actuators.

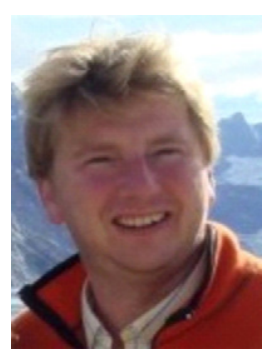

P.E. Sullivan received the B.S. and M.S. degrees in mechanical engineering from Clarkson University, Potsdam, NY, in 1988 and 1990, respectively, and the Ph.D. degree in mechanical engineering from Queen's University at Kingston, Kingston, ON, Canada, in 1995. In 1995, he joined the Department of Mechanical and Industrial Engineering, University of Toronto, Toronto, ON, where he is currently an Associate Professor. His interest is in the areas of microfluidics, turbulence related to aerodynamic control at low Reynolds numbers, and dusting control in rotating flows. 